\title{
The best plan of evacuation in Louvre
}

\author{
Yao Xiao ${ }^{1, a}$, Yinqiao Yan $^{2}$ and Yiqin Lei $^{3}$ \\ ${ }^{1}$ School of Mathematics and Statistics, Beijing Institute of Technology, China \\ ${ }^{2}$ Institute of Statistics and Big Data, Renmin University of China, China \\ ${ }^{3}$ Faculty of Science, Leiden University, Netherlands
}

\begin{abstract}
According to reports, since 2012, there have been 12 terrorist attacks in France. At the same time, the number of visitors of Louvre reached a peak of 8.1 million in 2017. Therefore, making an efficient evacuation plan in Louvre is particularly important. In our paper, we assume that the process of evacuation contains two main parts: the path from the starting point to the exit, and the location where the congestion may occur, such as stairs and exits. For the first part, we use the ant colony optimization algorithm to find the optimal path and get how many flows arrive at each exit. For the second part, as we already know the number of flows, we can use queuing theory to determine whether it is congested or not and calculate the corresponding congestion time. Finally, the optimal evacuation path and the corresponding time are obtained. In addition, associated with the application "Affluences" used in Louvre, some devices are designed to measure the average speed of the crowd movement. If the average speed is below a threshold value, an emergency exit will be opened to improve the evacuation process.
\end{abstract}

\section{Introduction}

The increasing number of terrorist attacks in France has led to the need of improving emergency evacuation plans for a variety of popular attractions, allowing visitors to evacuate the building quickly and efficiently in order to avoid casualties. The Louvre is one of the largest and most visited museums in the world, and it receives a huge number of visitors each year. In 2017, it received more than 8.1 million visitors. In addition, the unstable number of visitors in each day and the diversity of visitor types make evacuation in emergencies more challenging. Therefore, it is very important to establish a model and design an effective emergency management policy for the Louvre.

Due to the increasing number of terrorist attacks, the Louvre hopes to get a fast, effective and safe evacuation plan for visitors, so that once the emergency happens, the program can quickly evacuate all the staff and tourists and minimize casualties. We are required to help the supervisor develop an emergency evacuation model that allows staff to evacuate visitors while also allowing emergency personnel to enter the building as fast as they can. For this reason, we carry out two steps as follows: (i) Propose emergency management and procedures to the Louvre for the safety of tourists; (ii) Discuss how to apply the model to other large, crowded structures.

Notations. $T_{1}, T_{2}, T_{3}$ denote the disaster warning time, evacuation preparation time, and evacuation movement time respectively.

Assumptions. To simplify our model, we give some assumptions as follows without loss of generality. (i) The

\footnotetext{
a Corresponding author: xiaoyaoxy1998@sina.com
}

subway station does not consider the access restrictions in the event of a disaster. (ii) The roads are straight, regardless of the hindrance of the exhibits. (iii) All personnel fully obey the staff command and disabled go to dedicated channel. (iv) After the main export congestion, the crowd orderly queued out of the museum, which means once they start queuing, they no longer leave to other teams. (v) People in lower ground floor go to the Pyramid exit and the Richelieu exit; People in Napoleon hall are equivalent to not entering the museum; People in ground, 1st and 2nd floor are required to evacuation from ground floor. (vi) The elevator is out of service, only the stairs. (vii) Congestion may occur at the entrance and exit, other paths are smooth, therefore, other routes will not cause stamping accidents.

\section{Preliminaries}

In the case of special disasters (such as natural disasters or terrorist attacks), whether the people in the building can safely evacuate of people depends mainly on two characteristic times: First, the Available Safety Egress Time (ASET), which indicates the time required for the disaster to pose a danger to people. The second is the Required Safety Egress Time (RSET) [1], which is the time required for evacuating people to a safe area. If it can be ensured that the available safe evacuation time of all personnel in the building is greater than the safe evacuation time, the safety of the personnel in the building is guaranteed in the event of a disaster, which means the evacuation plan of the building is successful. 
Assuming the time from the occurrence of the disaster to the detection of the disaster by the indoor personnel is the disaster warning time $T_{1}$, the time taken for the staff to prepare for evacuation is the evacuation preparation time $T_{2}$, and the time for evacuating the visitors to reach the safety zone outside the building is the evacuation movement time $T_{3}$. So the required safety egress time $R S E T=T_{1}+T_{2}+T_{3}$ and $T_{2}$ are based on the specific situation. Then we will talk about $T_{3}$ in the following.

\subsection{Emergency evacuation route selection principle}

The optimal evacuation route is the best route for emergency evacuation. This route should ensure that the trapped people in the danger zone could be evacuated to safe places in the shortest time. Therefore, in combination with the question above, the best evacuation path should meet the following principles: (i) Try to minimize the time required for evacuation. (ii) Try to avoid the blocking exit. (iii) The evacuation path should have a clear indication. (iv) The building should have an alternate evacuation path.

\subsection{Basic theories of evacuation}

The basic research on evacuation is actually the study of various parameters in the evacuation process, including effective width, personnel density, personnel movement speed and personnel flow coefficient.

The speed of people flow, according to the literature [2], there is an empirical formula between the flow density and the speed of movement given by

$$
v=1.1 \rho^{-0.7954}(\mathrm{~m} / \mathrm{s}),
$$

where $v$ refers to the speed of personnel movement and $\rho$ refers to the personnel density.

The personnel flow coefficient refers to the number of people who can pass through the safety passage in unit width and unit time. In practice, it can be represented by the travel speed of the head of the flow. Under normal circumstances, when the flow coefficient of people is $N \leq$ 1.0 person $/\left(\mathrm{m} \cdot \mathrm{s}^{-1}\right)$, there is no delay of people in the safe evacuation passageway. At this time, the flow of people in the building is free [3].

According to the measured statistical data of the personnel flow coefficient, the reference value of the flow coefficient of the entrance and exit is shown in Table 1.

Table 1. Coefficient of personnel flow in channel or passageway.

\begin{tabular}{|c|c|c|}
\hline Flow state & $\begin{array}{c}\text { Type of entrance } \\
\text { and exit }\end{array}$ & $\begin{array}{c}\text { Personnel flow } \\
\text { coefficient } \\
\left.\text { (person } \cdot \mathrm{m}^{-1} \cdot \mathrm{s}^{-1}\right)\end{array}$ \\
\hline \multirow{2}{*}{$\begin{array}{c}\text { Emergency } \\
\text { evacuation }\end{array}$} & Corridor & 1.5 \\
\cline { 2 - 3 } & Stairwell entrance & 1.3 \\
\cline { 2 - 3 } & Refuge entrance & 1.5 \\
\hline
\end{tabular}

\subsection{The effect of disabled people}

The difference of the evacuation ability between disabled individual and healthy individual is the fundamental cause of the particularity of the evacuation of the disabled people. In emergencies, it is less likely that only a single disabled visitor in the Louvre, and disabled people will also hinder the evacuation efficiency in the stairs to some extent. In addition, the actual evacuation ability of disabled persons is affected by many external factors, including the external objective environment and other people in the surrounding area. Therefore, persons with disabilities should take emergency passages with the help of emergency personnel and do not evacuate with normal tourists, which can improve evacuation efficiency.

\section{Personnel evacuation optimization model}

Nowadays, models for solving emergency evacuation problem of large venues mainly include cellular automata [4], lattice gas model [5] and so on. These models mainly analyze the next evacuation path based on the current situation. However, they are not ideal in providing global optimization of individual space-time path analysis. Therefore, we carried out the following analysis.

In the emergency evacuation of the office building, the evacuation time $T_{3}$ can be divided into two parts: The first part is the movement time in the evacuation path $t_{31}$, including the leveling time and the stair time. Without severe blocking, moving forward at a certain speed can be guaranteed. And there is such a situation in which the queue is waiting long due to the small entrance and exit and the large flow of evacuated people. This refers to as the stagnant time $t_{32}$. Then we have $T_{3}=t_{31}+t_{32}$.

For the movement time $t_{31}$, since it is related to the path selection, the path optimization can shorten the time. Therefore it can be optimized by the adaptive ant colony optimization algorithm. For the stagnant time $t_{31}$, since it is the waiting time, so it can be optimized by queuing theory.

\subsection{Ant colony optimization algorithm}

Ant colony optimization algorithm is a bionic optimization algorithm used to find the optimal path in the graph. It was initially proposed by Marco Dorigo [6, 7], inspired by the behavior of ants finding the optimal path by releasing pheromones in searching for food. When solving large-scale complex problems, the effect of ant colony optimization is generally better than that of Floyd algorithm and Dijkstra algorithm due to the randomness, since the former has a certain probability to escape from local optima. Thus, the ant colony optimization algorithm is more robust.

Let $m$ be the number of ants, $n$ be the number of node, and $b_{i}(t)$ represents the number of ants at node $i$ at the time $t$. Then we have $m=\sum_{i=1}^{n} b_{i}(t) . V$ donates the set of 
nodes and $E$ denotes the set of edges between nodes. $\tau_{i j}(t)$ represents the information density at time $t$ on path $\langle i, j\rangle$ and $\Gamma=\left\{\tau_{i j}(t) \mid v_{i}, v_{j} \in V\right\}$ refers to the set of pheromone concentrations connecting all the nodes remaining on the path at the time $t$. The pheromone concentration is equal on each path at the initial time; the optimal path selection based on the ant colony optimization is looking for nodes in the directed graph $G=(V, E, \Gamma)$ to achieve the minimum cost of ownership.

During the movement of ant $k \in\{1,2, \cdots m\}$, the direction of the transfer is determined by the concentration of the pheromone on each path. Here, $t a b u_{k}$ is used to record the nodes that the ant $k$ is currently walking. The path set is dynamically adjusted with the evolution process. In the path selection process, the ants calculate the state transition probability according to the pheromone concentration on each path and the heuristic information of the path.

Let $p_{i j}^{k}(t)$ denote the state-transition probability with which the ant $k$ is transferred from node $i$ to node $j$ at the moment $t$. In [6], $p_{i j}^{k}(t)$ is defined by

$$
p_{i j}^{t}=\left\{\begin{array}{c}
\frac{\left(\tau_{i j}(t)^{\alpha}\right)\left(\eta_{i j}(t)^{\beta}\right)}{\sum_{s \in \text { allowed }_{k}}\left(\tau_{i s}(t)^{\alpha}\right)\left(\eta_{i s}(t)^{\beta}\right)}, j \in \text { allowed }_{k} \\
0, j \notin \text { allowed }_{k}
\end{array} .\right.
$$

In (1), allowed $_{k}=\{V$-tabu $\}$ indicates the node that the ant $k$ is allowed to select in the next step; $\alpha$ represents the information heuristic factor, reflecting the relative importance of the pheromone; $\beta$ represents the expected heuristic factor, reflecting the relative importance of the pheromone in the ant selection path during the movement; $\eta_{i j}(t)$ represents the local heuristic factor, reflecting the degree of expectation from the transition, usually taking the following expression $\eta_{i j}(t)=1 / d_{i j}$, where $d_{i j}$ denotes the Euclidean distance between two adjacent nodes $i$ and $j$. For ants $k$, the less $d_{i j}$ is, the larger $\eta_{i j}(t)$ will be, then the larger $p_{i j}^{k}(t)$ will be. Obviously, the heuristic function represents the degree to which the ant moves from the node $i$ to the node $j$.

After each ant has completed one step or completed the traversal of all nodes, the residual information is updated. As $\tau_{i j}(t)$ indicates pheromone concentrated on path $\langle i, j\rangle$ at moment $t$, we have

$$
\tau_{i j}(t+1)=(1-\rho) \tau_{i j}(t)+\sum_{k=1}^{m} \Delta \tau_{i j}^{k}(t)
$$

where $\rho$ is the pheromone volatilization coefficient and $\rho \in(0,1]$. 1- $\rho$ represents the pheromone concentration residual factor, indicating the relative importance of the residual pheromone. $\Delta \tau_{i j}(t)$ denotes the increment of pheromone concentration on path $\langle i, j\rangle$ between time $t$ and time $t+1$. In the initial moment $\Delta \tau_{i j}(t)=0$.

In different pheromone update strategies, the AntCycle model is better in global optimization because it utilizes global information. The update strategy is given by

$$
\Delta \tau_{i j}^{k}(t, t+n)= \begin{cases}\frac{Q}{L_{k}}, & (i, j) \text { on the path of ant } k \\ 0, & \text { otherwise }\end{cases}
$$

In (3), $Q$ represents the total concentration of pheromone released by each ant and $L_{k}$ represents the total tour length of ant $k$.

\subsection{Queuing theory}

A complete queuing system consists of input process, arrival rules, queuing rules, service organization structure, service time and service planning. The queuing system composition is given as follows.

The optimization problem, which is also called the system optimal design problem and the dynamic control problem, is one of the problems solved by the queuing theory. Queuing delay time can be optimized by queuing theory.

Average service time $T_{f}$. Average service time refers to the time when evacuation personnel pass the security exit, which is given by

$$
T_{f}=\frac{L}{V},
$$

where $L$ represents the length of the evacuation channel (the space connecting the internal exit and the outside world), and $V$ represents the speed at which the personnel evacuate the passage through the exit.

Average service rate $\mu$. The average service rate can be regarded as the average number of people served in a unit of time by the evacuation personnel, which is given by

$$
\mu=\frac{1}{T_{f}},
$$

Average arrival time $T_{d}$. The average arrival time is the number of evacuations divided by the number of people entering the waiting area by each route per unit time. It is given by

$$
T_{d}=\frac{M}{\sum_{i=1}^{n} N_{i} B_{i}}=\frac{\rho L W}{\sum_{i=1}^{n} N_{i} B_{i}},
$$

where $M$ is the number of people in the evacuation channel; $\rho$ is the population density; $L$ is the length of the evacuation channel for the exit; $W$ is the width of the evacuation channel for the exit; $n$ is the number of branches for the branch; $N_{i}$ is the flow for the entrance to branch $i$; and $B_{i}$ is the width of the entrance to branch $i$. 
Average arrival rate $\lambda$. The average arrival rate can be seen as the number of people arriving in a unit of time. It has the following form

$$
\lambda=\frac{1}{T_{d}} .
$$

\section{Implementation and results}

In this section, we will implement our personnel evacuation optimization model and give the numerical results including the optimal evacuation route and the correspondingly time. In the first part, we will demonstrate the results of applying ant colony optimization algorithm. In this case, The nodes represents all the exits and the position of stairs. Since people will not choose another stair if they already arrive at the position of one stair, we do not need to consider the length of stairs and a stair can be simplified as a node when we apply the ant colony optimization algorithm. In the second part, we will give the results of applying queuing theory. The places where queuing would happen in our model are the exits. Then we can obtain the total escaping time. All the results are conducted in MATLAB $\mathrm{R} 2017 \mathrm{~b}$ in Windows 10 on a Dell Laptop with an Intel(R) Core(TM) i7-5500U CPU at $2.40 \mathrm{GHz}$ and $8 \mathrm{~GB}$ of RAM.

Implementations. We assume that when a disaster occurs, people on the 1 st and 2 nd floors will choose the stairs closest to themselves to go downstairs. Therefore, the optimal escape route is such a path starting from the upper of the stairs and ending at the exit. Thus, the problem is transformed into the graph shown in Figure 1, starting from all the nodes at the 2 nd layer, and searching for the optimal path to each exit of the ground layer. Selecting the shortest time to use as the optimal escape route for that starting node.

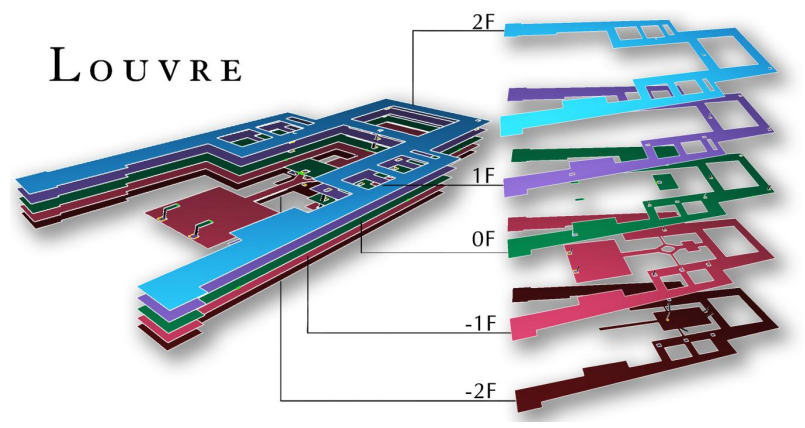

Figure 1. The building structure of Louvre.

\subsection{Results of ant colony optimization algorithm}

In this part, we will use the ant colony optimization algorithm to derive the optimal evacuation route and the movement time $t_{31}$. Parameters in this algorithm are set as $m=1, \alpha=1, \beta=5, \rho=0.1, k=50, Q=100$, and $v_{c o r}=$ $1.0 \mathrm{~m} / \mathrm{s}$. Based on the assumptions in Section 1, we just consider the tourists in the 1 st and 2 nd floor. We have numbered the stairs sequentially. The results are as follows in Table 2.
Table 2. The optimal evacuation routes and the movement time of the 1st and 2nd floor. St: Stair; Ri: Richelieu; Ar: Arts; Li: Lions.

\begin{tabular}{|c|c|c|}
\hline \multirow{2}{*}{ Floor } & Routes & Time \\
\hline \multirow{3}{*}{ 2nd } & St18 - St11 - Ri Exit 1 & 68.83 \\
\cline { 2 - 3 } & St19 - St13 - Ar Exit & 143.83 \\
\cline { 2 - 3 } & St20 - St16 - Ar Exit & 87.33 \\
\hline \multirow{7}{*}{ 1st } & St11 - Ri Exit 1 & 48.17 \\
\cline { 2 - 3 } & St12 - Ri Exit 1 & 140.67 \\
\cline { 2 - 3 } & St13 - Ar Exit & 123.67 \\
\cline { 2 - 3 } & St14 - Ar Exit & 113.67 \\
\cline { 2 - 3 } & St15 - Ar Exit & 102.67 \\
\cline { 2 - 3 } & St16 - Ar Exit & 66.67 \\
\cline { 2 - 3 } & St17 - Li Exit 2 & 48.67 \\
\hline
\end{tabular}

The visualized map of the evacuation routes are shown in Figure 2.

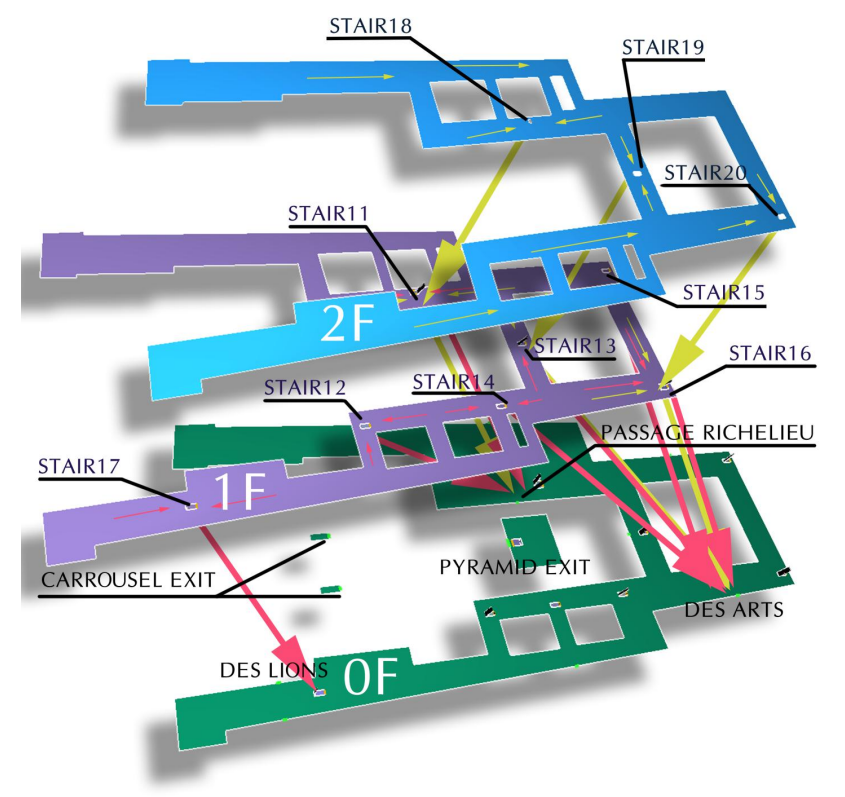

Figure 2. The visualized map of the evacuation routes of 1 st and 2 nd floor in Louvre.

\subsection{Results of queuing theory}

In this part, we will use the queuing theory to obtain the waiting time when congestion occurs. For queuing theory, we choose $L=10, V=0.6, W=1.2, N=1.3, B=0.8$, and $n=2$. We choose $\rho=1.0,1.5,2.0$ to test our model, representing low, medium and high population densities in practice respectively. The results of stairs and exits are as follows in Table 3 , Table 4 and Table 5 respectively.

Table 3. Congestion time at stairs. 


\begin{tabular}{|c|c|c|}
\hline & \multicolumn{2}{|c|}{$\rho$} \\
\hline \multirow{3}{*}{$\begin{array}{c}\text { Congestion } \\
\text { time }\left(\mathrm{s}^{-1}\right)\end{array}$} & 1.0 & 2.5 \\
\cline { 2 - 3 } & 275.33 & 439.82 \\
\hline
\end{tabular}

Table 4. Congestion situation at exits $(\rho=1.0)$. Br: Branches; Con: Congestion; Ri: Richelieu; Ar: Arts; Li: Lions; Py: Pyramid; Ca: Carrousel.

\begin{tabular}{|c|c|c|c|c|c|c|}
\hline Exits & $\mu$ & $\lambda$ & $\mathrm{Br}$ & Con & $\begin{array}{c}\text { Con } \\
\text { time }\end{array}$ & $\begin{array}{c}\text { Speed } \\
(\mathrm{m} / \mathrm{s})\end{array}$ \\
\hline $\mathrm{Ri}$ & 0.255 & 0.125 & 3 & $\mathrm{No}$ & - & 1.80 \\
\hline $\mathrm{Ar}$ & 0.500 & 0.333 & 6 & $\mathrm{No}$ & - & 2.40 \\
\hline $\mathrm{Li}$ & 0.500 & 0.055 & 1 & $\mathrm{No}$ & - & 0.40 \\
\hline $\mathrm{Py}$ & 0.167 & 0.074 & 2 & $\mathrm{No}$ & - & 0.40 \\
\hline $\mathrm{Ca}$ & 0.500 & 0.028 & 1 & $\mathrm{No}$ & - & 0.20 \\
\hline
\end{tabular}

Table 5. Congestion situation at exits $(\rho=2.5)$.

\begin{tabular}{|c|c|c|c|c|c|c|}
\hline Exits & $\mu$ & $\lambda$ & $\mathrm{Br}$ & Con & $\begin{array}{c}\text { Con } \\
\text { time }\end{array}$ & $\begin{array}{c}\text { Speed } \\
(\mathrm{m} / \mathrm{s})\end{array}$ \\
\hline $\mathrm{Ri}$ & 0.080 & 0.125 & 3 & $\mathrm{No}$ & - & 0.72 \\
\hline $\mathrm{Ar}$ & 0.160 & 0.333 & 6 & Yes & $\begin{array}{c}394 \\
83\end{array}$ & 0.96 \\
\hline $\mathrm{Li}$ & 0.160 & 0.055 & 1 & $\mathrm{No}$ & - & 0.16 \\
\hline $\mathrm{Py}$ & 0.053 & 0.074 & 2 & $\mathrm{No}$ & - & 0.16 \\
\hline $\mathrm{Ca}$ & 0.160 & 0.028 & 1 & $\mathrm{No}$ & - & 0.08 \\
\hline
\end{tabular}

The visualized evacuation situation at 60 seconds and 100 seconds are shown in Figure 3 and Figure 4.

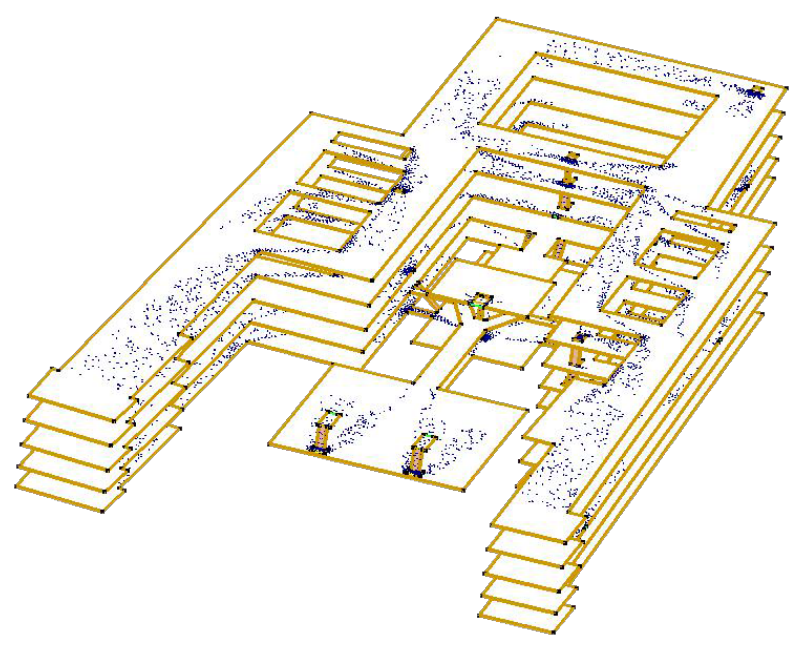

Figure 3. The visualized map of the evacuation routes of $1 \mathrm{st}$ and 2 nd floor in Louvre ( 60 seconds).

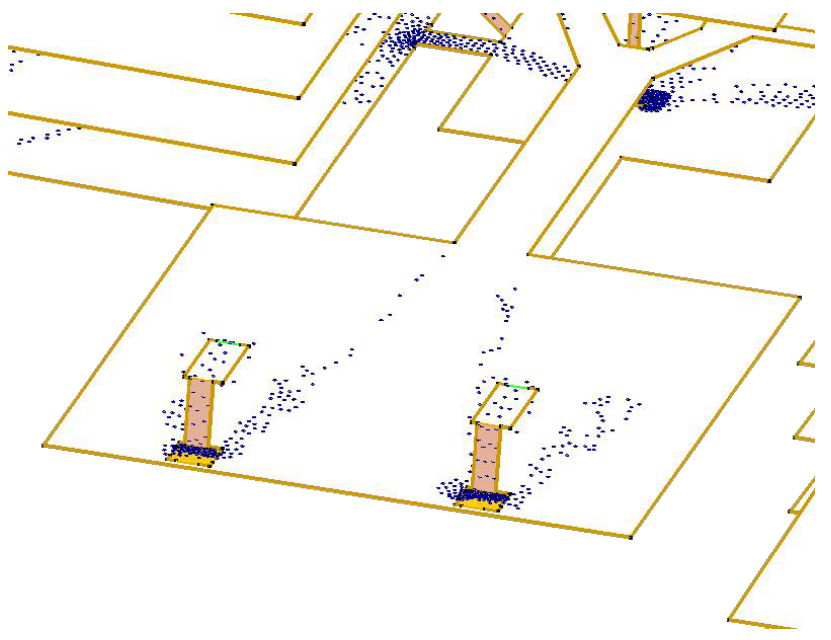

Figure 4. The visualized map of the evacuation routes of 1st and 2nd floor in Louvre (100 seconds).

We have the following observations.

(i) When $\rho=1.0$, the total escaping time is $148.5 \mathrm{~s}+$ $143.83 \mathrm{~s}+275.33 \mathrm{~s} * 2=14.05$ minutes. When $\rho=2.5$, the most distant coordinates on the second floor is $(457,192)$, 148.5 meters from the Stair19. After $148.5 \mathrm{~s}$, one will pass the route of Stair19-Stair13-Arts Exit. There is a congestion at the art exit and the congestion time is $43.13 \mathrm{~s}$. Therefore, the total escaping time is $148.5 \mathrm{~s}+$ $143.83 \mathrm{~s}+439.82 \mathrm{~s} * 2+394.83 \mathrm{~s}=26.11$ minutes.

(ii) The total time is then compared to the available evacuation time of the disaster. When the population density is small, it can ensure that everyone can successfully evacuate within the escaping time. When the population density is large, it can guarantee that the number of escapes is as much as possible within the escaping time.

\subsection{Emergency exit}

According to the assumptions, in the case of high population density, only one emergency door is allowed to open. We implement our model again and see that the evacuation effectiveness is the best when the Emergency Door 1 is opened. The results are as follows in Table 6 and Table 7.

Table 6. The optimal evacuation routes and the movement time of the 1st and 2nd floor when emergency exit 1 is opened. Ri: Richelieu; Ar: Arts; Li: Lions; Em: Emergency.

\begin{tabular}{|c|c|c|}
\hline \multirow{2}{*}{ Floor } & Routes & Time \\
\hline \multirow{3}{*}{ 2nd } & St18 - St11 - Ri Exit 1 & 68.83 \\
\cline { 2 - 3 } & St19 - St13 - Em Exit 1 & 62.59 \\
\cline { 2 - 3 } & St20 - St16 - Ar Exit & 87.33 \\
\hline \multirow{3}{*}{ 1st } & St11 - Ri Exit 1 & 48.17 \\
\cline { 2 - 3 } & St12 - Ri Exit 1 & 140.67 \\
\cline { 2 - 3 } & St13 - Em Exit 1 & 44.11 \\
\hline
\end{tabular}




\begin{tabular}{|c|c|c|}
\hline \multirow{2}{*}{ St14 - Em Exit 1 } & 107.67 \\
\cline { 2 - 3 } & St15 - Ar Exit & 102.67 \\
\cline { 2 - 3 } & St16 - Ar Exit & 66.67 \\
\cline { 2 - 3 } & St17 - Li Exit 2 & 48.67 \\
\hline
\end{tabular}

Table 7. Congestion situation at exits $(\rho=1.5)$. Congestion situation at exits. Ri: Richelieu; Ar: Arts; Li: Lions; Py: Pyramid; Ca: Carrousel.

\begin{tabular}{|c|c|c|c|c|c|c|}
\hline Exits & $\mu$ & $\lambda$ & $\mathrm{Br}$ & $\mathrm{Con}$ & $\begin{array}{c}\text { Con } \\
\text { time }\end{array}$ & $\begin{array}{c}\text { Speed } \\
(\mathrm{m} / \mathrm{s})\end{array}$ \\
\hline $\mathrm{Ri}$ & 0.12 & 0062 & 3 & $\mathrm{No}$ & - & 0.60 \\
\hline $\mathrm{Ar}$ & 0.24 & 0.167 & 6 & $\mathrm{No}$ & - & 0.80 \\
\hline $\mathrm{Li}$ & 0.24 & 0.028 & 1 & $\mathrm{No}$ & - & 0.13 \\
\hline $\mathrm{Py}$ & 0.08 & 0.037 & 2 & $\mathrm{No}$ & - & 0.13 \\
\hline $\mathrm{Ca}$ & 0.24 & 0.014 & 1 & $\mathrm{No}$ & - & 0.07 \\
\hline
\end{tabular}

The farthest point from the lion exist is on the first floor, $148.5 \mathrm{~m}$ from the exist, speed $1 \mathrm{~m} / \mathrm{s}, 148.5 \mathrm{~s}$, and arrive at the lion exist through Stair12 - Lion Exit. Also the exit is not congested.

The final evacuation time: $148.5 \mathrm{~s}+140.67 \mathrm{~s}+439.82 \mathrm{~s}$ $* 2=22.43$ minutes $<26.11$ minutes. It can be seen that after opening the Emergency Exist 1, it provides a good diversion effect for the art door, and successfully solved the art door congestion.

\subsection{Sensitivity analysis}

\subsubsection{Characteristics of basic ant colony optimization parameter}

Many scholars have discussed the parameters of the basic ant colony optimization algorithm. The parameters $Q, C$, $\alpha, \beta$, and $\rho$ can be determined the optimal combination by experimental methods. There is no theoretical basis at present, and the published results are for specific problems. For the classic TSP, the empirical result $0 \leq \alpha$ $\leq 5,1 \leq \beta \leq 5, Q$ has little effect and $C$ select relative set based on the fitness of the problem.

\subsubsection{Selection of pheromone volatility}

In the ant colony optimization, the artificial ant has the function of human memory. As the time pass, the information left before will gradually disappear, and the parameter 1- $\rho$ indicates the disappear degree in the algorithm model. $\rho$ is the signal residual coefficient. Ant colony optimization, like genetic algorithm and other simulation evolution algorithms, also has the disadvantages of slow convergence speed and easy to fall into local optimum. The amount of information volatility $1-\rho$ is directly related to the global search ability of ant colony optimization and its convergence speed. Due to the existence of information volatility, when the problem is large, it will make the amount of information in those paths that have never been searched reduce to 0 , thus reducing the global search ability of the algorithm, and when $1-\rho$ is too large, the previously searched path is too likely to be selected again, which will also affect the random performance and global search ability of the algorithm. Conversely, by reducing the amount of information volatility $1-\rho$, although it can improve the random performance and global search ability of the algorithm, the convergence speed of the algorithm is reduced.

\section{Conclusion}

In this paper, an evacuation model based on the combination of adaptive ant colony optimization algorithm and queuing theory has been proposed as an efficient tool to help the staff in Louvre to solve the difficult escape problem. Our model has the characteristics of information positive feedback and heuristic search, which guarantees that the model can not only solve the evacuation problems under the circumstances of non-specific-location occurrences such as terrorist attacks, but also solve the problems with specific-location occurrences such as fires and stampedes. For the latter case, all we need to do is to cut off the paths where the disaster occurs when applying the ant colony optimization algorithm. Thus, it can design the best escape route of specific disasters.

We also provide the staff with some suggestions:

- Emergency personnel should use emergency exits that are not open during evacuation. When entering the building, their duty is to help the elderly and children to evacuate, and to explain the route for foreign tourists;

- The application "Affluences" can be used as a speed measuring instrument during evacuation. When it shows that a certain exit is blocked, the staff should immediately open the Emergency Exit 1 and guide the visitors to evacuate based on the new evacuation route.

\section{References}

1. C. Wentao, X. Dan, Journal of Shenyang Aerospace University, 4, 76-79 (2011).

2. F. Liu, S. Liao, Chongqing: Chongqing University Press (2007).

3. D. Wu, S. Yang. China Safety Science Journal, 22, 56-62 (2012).

4. W. Yuan, K.H. Tan, Physica A. Statistical Mechanics and Its Applications, 384, 549-566 (2007).

5. W. Zhang, L. Zhao, Journal of Safety and Environment, 10, 169-172 (2010).

6. M. Dorigo, V. Maniezzo, A. Colorni, IEEE Trans. Syst. Man Cybern Part B-Cybern, 26, 29-41 (1996).

7. M. Dorigo, $\mathrm{PhD}$ thesis, Politecnico di Milano, Italy (1992). 